Revue des patrimoines

43 | 2021

Des écoles d'art académiques aux écoles d'art : des collections et des lieux, un patrimoine à valoriser

\title{
Les collections de l'École de dessin de Montpellier (1779-1825)
}

\section{Un patrimoine effacé}

The École de dessin de Montpellier's collections (1779-1825). An erased heritage

\section{Pierre Stépanoff}

\section{(2) OpenEdition Journals}

Édition électronique

URL : http://journals.openedition.org/insitu/29088

DOI : 10.4000/insitu.29088

ISSN : $1630-7305$

\section{Éditeur}

Ministère de la Culture

\section{Référence électronique}

Pierre Stépanoff, « Les collections de l'École de dessin de Montpellier (1779-1825) », In Situ [En ligne], 43 | 2021, document 11, mis en ligne le 12 janvier 2021, consulté le 14 janvier 2021. URL : http:// journals.openedition.org/insitu/29088; DOl : https://doi.org/10.4000/insitu.29088

Ce document a été généré automatiquement le 14 janvier 2021.

\section{c) $(9)$}

In Situ Revues des patrimoines est mis à disposition selon les termes de la licence Creative Commons Attribution - Pas d'Utilisation Commerciale - Pas de Modification 4.0 International. 


\title{
Les collections de l'École de dessin de Montpellier (1779-1825)
}

\author{
Un patrimoine effacé \\ The École de dessin de Montpellier's collections (1779-1825). An erased heritage
}

Pierre Stépanoff

En 1825, le peintre François-Xavier Fabre (1766-1837), né à Montpellier en 1766 et installé à Florence depuis 1793, offre à sa ville natale un important ensemble de peintures, sculptures et dessins, à charge pour le conseil municipal de lancer la construction d'un musée dédié à la présentation de cette collection. Le musée Fabre ouvre ses portes en 1828, dans l'ancien hôtel de Massilian, remanié selon le goût et les plans de son fondateur. Sur la rue Henri-IV, actuelle rue Montpelliéret, la façade du musée évoque les attaches florentines de Fabre, avec ses consoles, ses fenêtres thermales et son appareil de pierre aux lignes de refend bien marquées, dans l'esprit des palazzi de la Renaissance [fig. 1]. Fabre en est le directeur jusqu'à sa mort, en 1837. Les deux autres principaux donateurs de l'établissement, les collectionneurs Antoine Valedau (1777-1836) et Alfred Bruyas (1821-1877), placent leurs dons et legs dans la lignée du geste généreux de Fabre, dessinant de cette manière le récit du musée de Montpellier, tel qu'il s'énonce encore aujourd'hui ${ }^{1}$, marqué par des enrichissements successifs, fruits de la libéralité de personnes privées. 
Figure 1

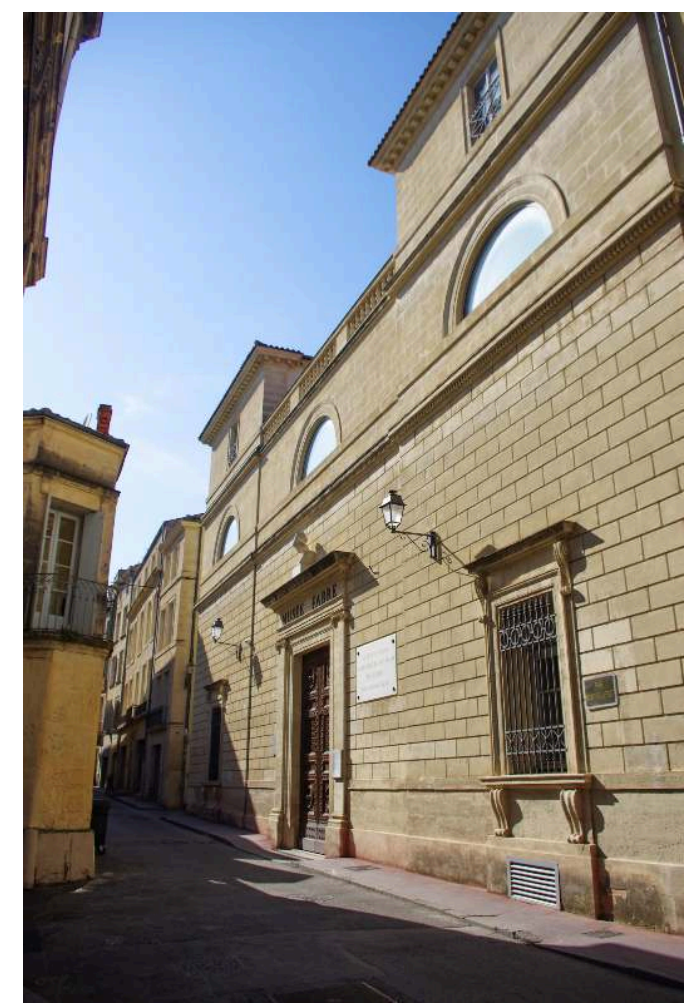

Ancienne entrée du musée Fabre (1825-1828), rue Montpelliéret, Montpellier.

(c) Photo Pierre Stépanoff, 2020.

2 Ce récit occulte cependant l'origine plus ancienne du musée de Montpellier. Le 11 août 1795, un premier établissement, certes modeste, était fondé sur ordre du directoire du département, prenant le nom de Muséum de l'Hérault. Selon les préconisations du pouvoir central, l'établissement se voyait confier la garde des tableaux et objets d'art saisis dans les établissements religieux ou les collections des émigrés, dans l'attente de l'aménagement et de l'ouverture d'un local digne de ce nom. Installé dans l'ancien collège des Jésuites (XvII ${ }^{\mathrm{e}}$.) [fig. 2], par où se fait l'entrée dans le musée Fabre depuis les travaux des années 2002-2007, l'établissement, essentiellement un dépôt d'œuvres d'art, partage ses murs avec l'école centrale du département. Ces institutions sont ellesmêmes les héritières de la Société des beaux-arts de Montpellier. Créée en 1779 par un groupe de trente personnes, administrateurs, ecclésiastiques, membres de la noblesse comme de la bourgeoisie du Languedoc et de Montpellier, cette société, qui se réunit dans ledit collège des Jésuites, se donne pour objectif d'encourager la formation artistique au moyen de la création d'une école gratuite de dessin et de développer le goût des arts auprès du public en y organisant des salons ${ }^{2}$. Au fil des ans, une collection de moulages en plâtre et de modèles dessinés ou gravés se constitue, grâce aux efforts du marchand d'art Abraham Fontanel (1741-1817), principal initiateur et animateur de la Société, nommé garde des plâtres et modèles de l'institution ${ }^{3}$. Cette histoire à la fois méconnue et déterminante pour la genèse du musée et le cadre de la vie artistique moderne dans la ville de Montpellier mérite que l'on s'y attarde. 


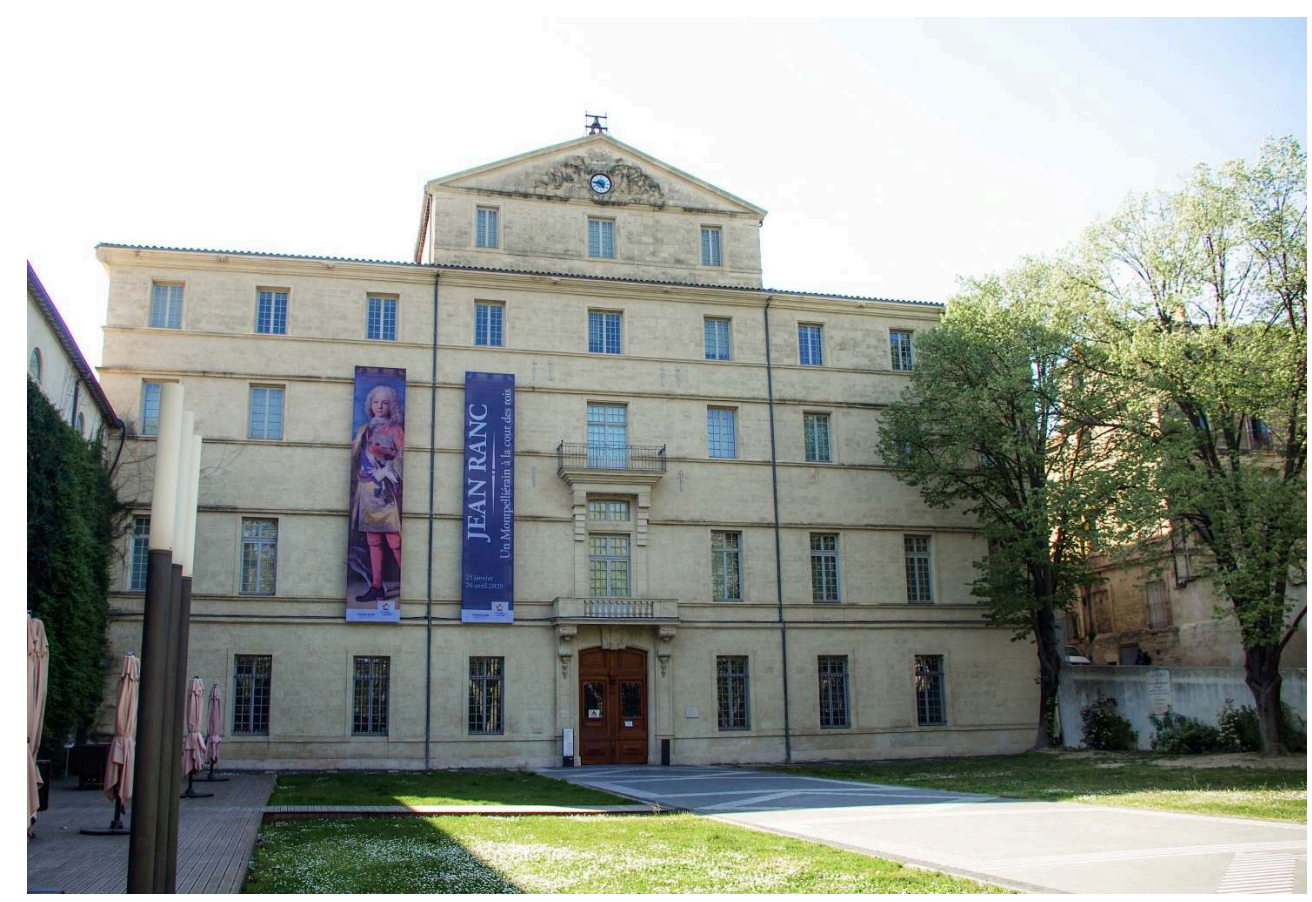

Collège des Jésuites, troisième quart du xvII ${ }^{\mathrm{e}}$ siècle, actuelle entrée du musée Fabre, Montpellier.

\section{La collection de l'École de dessin de Montpellier (1787-1825) : un enseignement fondé sur la copie de modèles}

C'est le 18 janvier 1779 que la Société des beaux-arts de Montpellier voit le jour, entraînant la création d'une école gratuite de dessin qui ouvre ses portes le 20 avril. À la fin de l'année, le 28 décembre, le premier salon de peinture, sculpture et gravure ouvre ses portes au public montpelliérain ${ }^{4}$. Ce projet s'inscrit pleinement dans le phénomène de diffusion, à travers la France et l'Europe, du modèle académique. À Montpellier, durant les huit années d'existence de la Société des beaux-arts, des cours de dessin sont prodigués sans discontinuer, à raison de deux heures par jour, tous les jours. Selon le modèle classique défini dès le xvir siècle par l'Académie royale de peinture et de sculpture à Paris et dès le $\mathrm{xvI}^{\mathrm{e}}$ siècle par les diverses académies italiennes, l'enseignement artistique repose sur la pratique de la copie: les élèves doivent s'imprégner des modèles, antiques ou modernes, reconnus comme des classiques, en les dessinant. C'est dans la pratique de la copie que se résume l'essentiel des leçons de dessin dispensées deux heures par jour dans les classes de la Société des beaux-arts ${ }^{5}$.

\section{La collection de l'École gratuite de dessin (1779-1787)}

Cette pratique de la copie, placée au cœur de l'enseignement, rend décisif le rôle d'Abraham Fontanel, garde des plâtres et modèles, chargé de constituer une collection 
de matériel pédagogique disponible à l'usage des maîtres comme des élèves. Ce personnage, installé à Montpellier depuis 1772, est à la tête d'un commerce d'art dans la ville et possède de solides relations avec de nombreux artistes et marchands de la capitale [fig. 3]. Son rôle est défini à l'article 17 des statuts et règlements de la Société des beaux-arts :

Un des associés domiciliés sera chargé, sur un inventaire fait double et signé du President et dudit associé domicilié, de la garde des platres, estampes et desseins appartenant à la Société. Il s'obligera de representer lesdits platres, estampes et dessein - En tout ou en partie ${ }^{6}$.

Figure 3

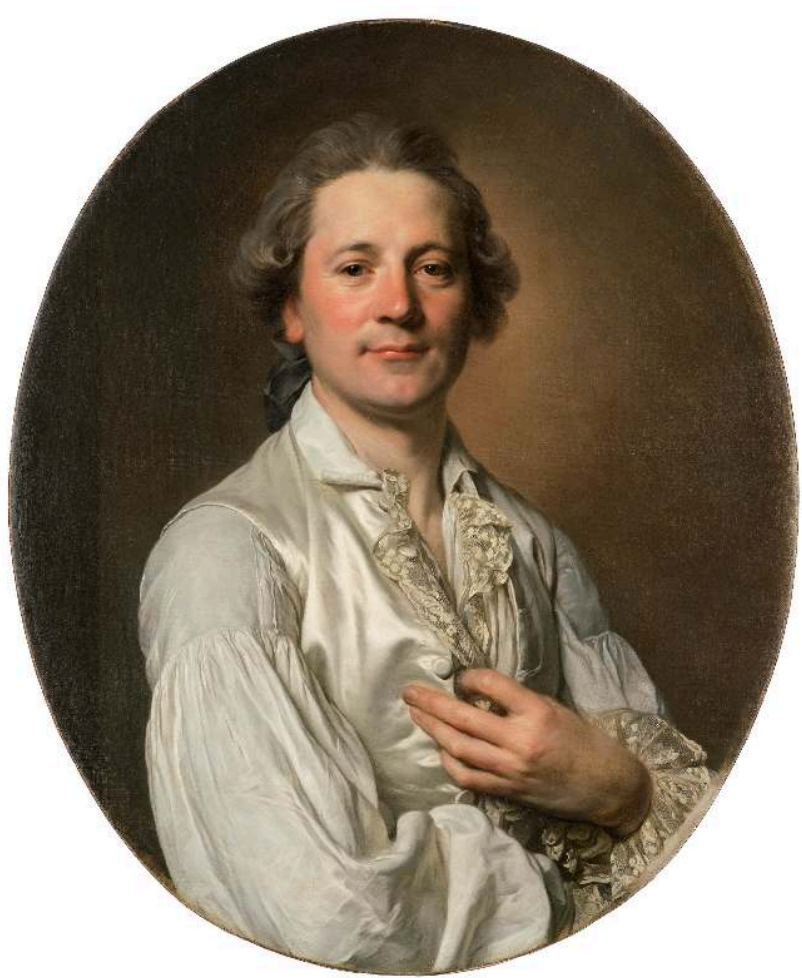

Portrait d'Abraham Fontanel (1741-1817), Joseph Siffred Duplessis, vers 1779, huile sur toile conservée à l'église des Pénitents bleus, Montpellier, classée Monument historique le 2 mai 2005.

Reproduction Frédéric Jaulmes, 2017 (Montpellier - Méditerranée Métropole).

5 Abraham Fontanel fait ainsi jouer ses relations avec le sculpteur Jean-Antoine Houdon (1741-1828) afin de lui acheter un «Grand Écorché ${ }^{~ » . ~ H o u d o n ~ o f f r e ~ d a n s ~ l e ~ m e ̂ m e ~ t e m p s ~}$ à la Société un buste de Molière en plâtre ${ }^{8}$ [fig. 4] ainsi qu'une tête de mort et un grand pied moulés, également en plâtre ${ }^{9}$. Cet enrichissement de la collection se poursuit deux mois plus tard. Lors de la séance du 18 avril 1779, les sociétaires décident d'acquérir pour 28 livres un ensemble de " parties détachées d'étude en plâtre pour distribuer aux élèves de l'école de la ronde bosse ", à savoir "sept parties doubles de bras, et des mains d'hommes avec des mains de femmes qui ont été moulées sur la nature ${ }^{10} »$. Quelques mois plus tard, le sculpteur Clodion (1738-1814) est reçu membre artiste de la Société des beaux-arts. Lors de la séance du 31 octobre 1780, Jean Antoine du Vidal, marquis de Montferrier, annonce qu'il remet deux plâtres offerts par le sculpteur pour son agrément en qualité de membre de la société: le Jupiter tonnant et le Fleuve Scamandre ${ }^{11}$. Dans le même temps, le marquis de Montferrier offre un Hercule Farnèse de 
27 pouces $(e n v .70 \mathrm{~cm}$ ) et un buste d'amour, exécutés par Pierre-Étienne Monnot (1657-1733).

Figure 4

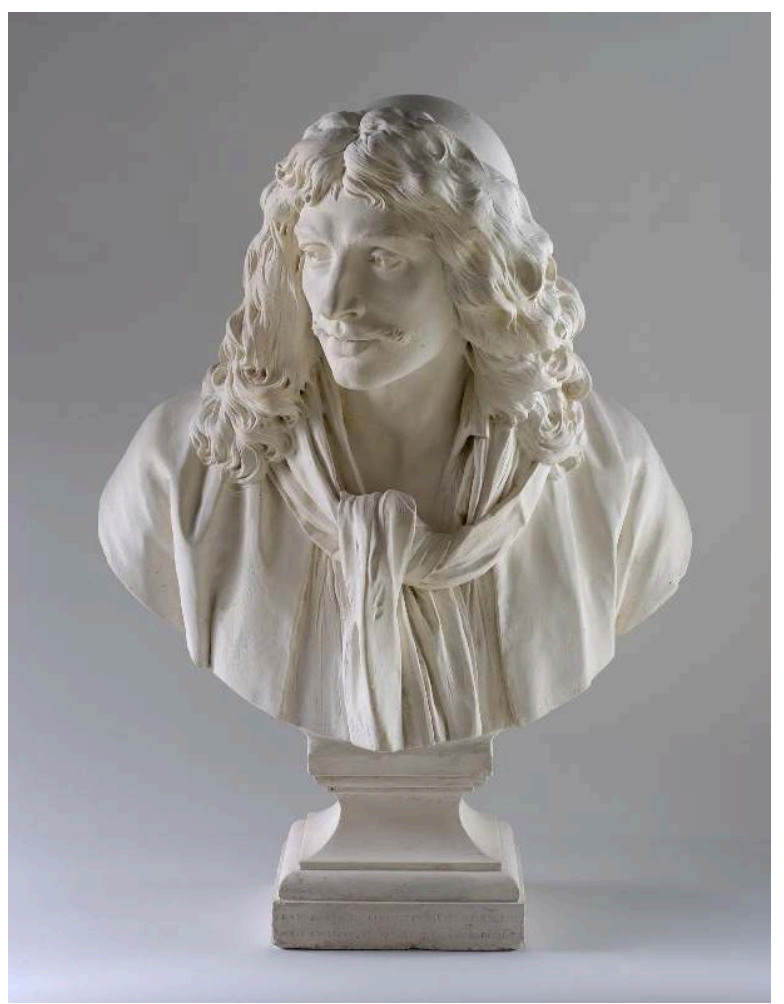

Molière, Jean-Antoine Houdon, vers 1778, plâtre conservé au musée Fabre (inv. 806.31), Montpellier. (c) Photo Frédéric Jaulmes, 2017 (Montpellier - Méditerranée Métropole)

Mais c'est en 1782 que la collection reçoit son enrichissement le plus considérable, grâce à une occasion saisie par Fontanel sur le port de Marseille. Cette acquisition est rapportée à la fois par les registres de la Société des beaux-arts et un mémoire rédigé par Fontanel en $1796^{12}$. Lors de la séance du 6 juin 1782, la société acte en effet l'achat d'un ensemble de trente-huit têtes, moulées d'après l'antique, repéré par Fontanel en provenance d'Italie. Cet ensemble de sculptures est détaillé dans l'inventaire des biens de la Société des beaux-arts dressé par le garde des plâtres quelques années plus tard, lors de sa dissolution en $1787^{13}$ : un Ajax, une Minerve, un Caracalla, un Rémouleur, un Zénon, un Apollon, un Marc-Aurèle, un Socrate, une Ariane, un Antinoüs, un César, un Moïse d'après Michel-Ange et d'autres modèles non identifiés.

Les documents relatifs à l'histoire de la Société des beaux-arts ne nous renseignent pas sur les circonstances d'autres acquisitions. Cependant, l'inventaire de 1787 lors de la transformation de l'école de dessin en une école des Ponts et Chaussées financée par les deniers publics permet de connaître l'intégralité de la collection accumulée durant ces sept années. Outre les sculptures de Houdon, de Clodion, de Monnot ainsi que les 38 têtes achetées à Marseille, on apprend ainsi que les élèves ont à leur disposition "soixante-quatre modèles collés sur quarton" ainsi que 64 autres modèles représentant des "grosses et moyennes têtes", 86 modèles figurant "différents principes d'ornement » et "quarante-sept académies entières ». Dans la collection de plâtres est également mentionné, sans que l'on en connaisse la provenance, un buste de 
Jean Deydé (1617-1687), l'arrière-grand-père d'un des membres de la Société, Bernard Daniel Deydé (1750-1793) ${ }^{14}$. On retrouve également une tête de Vierge et « une autre petite tête d'après Puget». Cet ensemble regroupe donc, en 1787, 261 feuilles et 68 sculptures à la disposition des élèves.

\section{La collection de l'École des arts, ponts et chaussées, de l'École centrale puis de l'École municipale (1787-1825)}

8 Le 19 janvier 1787, les États de Languedoc rattachent la Société des beaux-arts à une nouvelle École des arts, ponts et chaussées, qu'ils financent. Les locaux, le personnel et l'organisation des classes restent les mêmes. C'est à l'occasion de ce changement de statut qu'Abraham Fontanel rédige l'inventaire déjà mentionné des quelques tableaux, du matériel pédagogique, estampes et plâtres, ainsi que du mobilier dont la propriété est transférée à cette nouvelle école.

Durant la Révolution, l'École des arts, ponts et chaussées, devenue École centrale du département, joue un rôle décisif dans l'apparition du premier musée de Montpellier, poursuivant ainsi cette logique de constitution d'une collection. Le directoire du département de l'Hérault fonde le 11 août 1795 le Muséum du département. Les locaux de l'école servent ainsi de dépôt pour les œuvres d'art saisies dans les établissements religieux ou les collections des émigrés et c'est son personnel, en particulier Fontanel mais aussi le peintre Jacques Bestieu ${ }^{15}$ (1754-1842), directeur de l'École de dessin depuis 1786, qui sont chargés de réaliser ces saisies, d'en estimer la valeur et de veiller à la conservation des œuvres d'art. Outre les saisies révolutionnaires, la collection de modèles est également enrichie par l'acquisition, en août 1795, d'un ensemble de plâtres de la collection de Philippe-Laurent de Joubert (1729-1792), décédé trois ans plus tôt. Joubert, trésorier de la Bourse des États de Languedoc et membre fondateur de la Société des beaux-arts de Montpellier, était incontestablement le plus grand collectionneur de cette assemblée ${ }^{16}$. Si son cabinet de peintures se trouvait dans son hôtel particulier parisien place Vendôme, Joubert possédait également à Montpellier un bel ensemble de reproductions de sculptures antiques, dont l'Administration centrale du département de l'Hérault fait l'acquisition pour le musée et dont Fontanel rédige l'inventaire en mars $1796^{17}$. Ce lot comprend une quinzaine de têtes moulées d'après l'antique, notamment celle de la Vénus Médicis, du Bacchus [Dionysos] Sardanapale, de Vitellius, empereur romain. La collection possédait un grand intérêt archéologique puisqu'elle comprenait également de nombreux bas-reliefs, frises, urnes et autels moulés. Les moulages des deux Centaures de Furietti, d'un écorché de Bouchardon et d'un autre d'après nature, ainsi que celui d'un consul sur sa chaise curule et de son épouse, devaient constituer les pièces les plus spectaculaires de cet ensemble.

Un nouvel inventaire, rédigé en décembre 1797, lors de la passation de pouvoir entre Abraham Fontanel, limogé, et son successeur Claude Daumas, permet de connaître l'ensemble de la collection ${ }^{18}$, enrichie par l'achat de la collection Joubert, à laquelle vient s'ajouter, en dernier item, un moulage d'après le Gladiateur Borghèse exécuté et offert par le sculpteur actif à Marseille Étienne d'Antoine (1737-1809), auteur, quelques années plus tôt, de la Fontaine des licornes et du groupe des Trois Grâces, deux commandes de la Ville de Montpellier. 


\section{Le devenir de cette collection, de 1825 à nos jours. L'effacement d'une collection pédagogique}

11 Au fil des années d'existence de ces différentes institutions pédagogiques, la collection n'a cessé de s'enrichir. Alors que la notion de musée commence à apparaître à Montpellier sous la Révolution, la conception d'un matériel pédagogique perçu comme une collection à préserver dans l'intérêt des élèves était déjà clairement définie dix ans plus tôt. En effet, lors de la séance du 19 avril 1781, les membres de la Société des beaux-arts avaient déjà inscrit le principe d'inaliénabilité de cette collection :

Tous les effets quelconques appartenant actuellement à la Société ou qu'elle pourrait avoir dans la suite, soit qu'il lui ayent été donnés par quelqu'un de MM. les associés, ou par quelque amateur, ou qu'elle en ait fait elle-même la dépense et l'acquisition, appartiendraient en toute propriété au corps de la Société sans qu'aucun de MM. les associés, soit qu'il vint à quitter la ville de Montpellier, ou qu'il ne voulut plus être du nombre de MM. les associés fondateur après l'expiration du terme des trois premières années de leur engagement puisse sous aucun prétexte rien prétendre ni demander sur les dits effets [...] de manière que les effets puissent toujours être assurés au corps de la Société ${ }^{19}[. .$.$] .$

Le mode de rédaction des différents inventaires, aussi bien en 1787 qu'en 1797, révèle une absence de distinction entre d'une part les œuvres d'art originales, qu'il s'agisse des tableaux issus de la collection de la Société des beaux-arts (notamment un Bacchus et Ariane [Ariane dans l'île de Naxos] de Jean-François de Troy ${ }^{20}$ ), des saisies révolutionnaires (le Saint Jean Baptiste du Montpelliérain Joseph-Marie Vien, par exemple ${ }^{21}$ ) et d'autre part les ensembles de plâtres et de gravures utilisés pour l'enseignement. Les œuvres ne possèdent alors aucun numéro d'inventaire et se retrouvent énoncées, page après page, dans un seul et même document ne distinguant pas les collections patrimoniales du musée révolutionnaire et le matériel pédagogique de l'école de dessin. Cette absence de distinction ne doit pas surprendre, à une époque où la vocation première des musées est l'éducation des citoyens et des artistes par la fréquentation des chefs-d'œuvre de l'art. À cet égard, la conservation et l'acquisition de copies d'antiques aux côtés d'œuvres originales n'impliquent aucune contradiction.

Même si le musée de Montpellier voit son existence sanctionnée d'un point de vue juridique dès 1795 , il faut attendre 1811 pour que l'établissement soit ouvert au public. Un inventaire, rédigé par Jacques Bestieu en $1806^{22}$, nous permet de connaître l'état de la collection de plâtres depuis le précédent inventaire rédigé près de dix ans plus tôt, en 1797. Cet inventaire atteste de la bonne conservation de la collection, que l'on retrouve intégralement, item par item, dans les deux inventaires, notamment l'Écorché au bras relevé de Houdon, les deux Centaures, les plâtres de Clodion, de Monnot ou de d'Antoine. En 1825, la refondation par François-Xavier Fabre (1766-1837) du musée de la Ville de Montpellier et son ouverture, en 1828, changent considérablement le statut de cet ensemble. Les œuvres présentées au public, salle par salle, nous sont connues par les différents catalogues publiés du vivant de Fabre (1828 et 1830) et font office d'aide à la visite. Ces catalogues ne constituent pas un inventaire complet des collections et ne mentionnent que quatre plâtres modernes, donnés par les sculpteurs Jean-Baptiste de Bay (1779-1863) et Jean-François Legendre-Héral (1796-1851)23. L'inventaire Desmazes est à cet égard un document plus précis. Il s'agit du premier catalogue complet des collections du musée, rédigé par Casimir Desmazes (1800-1876), receveur 
municipal, l'année de la mort de Fabre. L'inventaire décrit ainsi les plâtres présents dans les salles, au-delà des quatre sculptures mentionnées ci-dessus, ce que ne faisaient ni le catalogue de 1828 ni celui de 1830 . On apprend ainsi qu'un certain nombre d'entre eux ont été donnés en 1825 ou légués en 1837 par Fabre, notamment des têtes, des bustes et des bas-reliefs. On retrouve également la liste partielle de nos plâtres issus de la collection de la Société des beaux-arts et du musée révolutionnaire ${ }^{24}$ : c'est le cas du Gladiateur Borghèse, du Jupiter de Clodion, des deux Centaures, de neuf têtes et bustes ainsi que de huit bas-reliefs. Une observation, ajoutée à la fin de cette liste, précise le sort des autres moulages: "Il existe à l'école de peinture une grande quantité de plâtres que la ville possédait avant l'établissement du musée ou que Mr. Fabre lui a donnés. Ils n'ont pas été inventoriés. Tous ceux compris et détaillés dans le présent inventaire se trouvent dans les galeries et l'escalier du musée. » La distinction entre collection patrimoniale et matériel pédagogique est désormais clairement établie. Fabre, ancien élève de l'école gratuite de dessin de Montpellier, avait été soucieux de créer une école de dessin ainsi qu'une bibliothèque dans les murs mêmes du musée Fabre. Cette triple fondation a également eu pour conséquence une répartition plus claire des différents objets dans les différents espaces, selon leur fonction, entre formation et délectation. On peut ainsi présumer que ce sont les plâtres les plus spectaculaires et qui présentaient le meilleur état de conservation qui sont venus scander le parcours de visite du musée.

Cette répartition, ainsi que la fragilité du matériau, a été fatale à cet ensemble. Après l'inventaire Desmazes, les multiples rééditions du catalogue de François-Xavier Fabre $(1839,1843,1850,1859,1866)$ ne mentionnent plus les différents plâtres accumulés au fil du temps par la Société des beaux-arts et sous la Révolution. Cette disparition de tout un pan de la collection contribue à occulter considérablement les trente années du musée primitif de Montpellier, de 1795 à 1825. Aujourd'hui cependant, les recherches sur les fonds du musée permettent de retrouver çà et là quelques éléments issus de cette collection.

Les deux premières acquisitions réalisées par la Société des beaux-arts, dès 1779, l'Écorché au bras relevé et le buste de Molière de Jean-Antoine Houdon, ont été conservées. Le Molière a reçu au XIX siècle une patine imitant le bronze qui a finalement été retirée au $\mathrm{Xx}^{\mathrm{e}}$ siècle pour redonner à l'œuvre un aspect plus fidèle à celui d'origine. Acquise grâce à un don d'artiste dans une cité ayant préservé le souvenir, dans le bâtiment même de l'hôtel de Massilian, d'une représentation théâtrale donnée par Molière lui-même, cette œuvre est pour ainsi dire la toute première des collections du musée, avant même que le musée n'ait existé. Elle entre aujourd'hui en dialogue harmonieux avec le riche fonds d'œuvres de Jean-Antoine Houdon du musée Fabre, notamment avec les sculptures ayant appartenu en propre à Abraham Fontanel (un buste de Benjamin Franklin ${ }^{25}$ en plâtre et un Voltaire assis ${ }^{26}$ en terre cuite), celles ayant été exposées au Salon de la Société des beaux-arts avant d'être acquises par le directoire du département (bustes de Voltaire, de Rousseau, en marbre ${ }^{27}$ ), celles offertes par la suite par d'autres donateurs (les figures de L'Été et de L'Hiver, données par le baron Auguste Creuzé de Lesser peu après l'ouverture du musée Fabre en $1828^{28}$ ) et les acquisitions plus récentes (une esquisse en terre cuite de L'Hiver, acquise en $1995^{29}$ ). S'il existe plusieurs plâtres de Molière tirés par Houdon lui-même, la précision des connaissances relatives à ce tirage, les circonstances bien documentées de son don lui confèrent un intérêt tout particulier. Le musée Fabre conserve un autre Écorché [fig. 5], moulé 
d'après le modèle conçu par Houdon en 1790 et dont la date d'arrivée au musée n'est pas connue, mais qui se confond peut-être avec le second Écorché mentionné dans l'inventaire de 1806. Son histoire est plus difficile à élucider ${ }^{30}$. L'œuvre, selon la documentation du musée, a en effet rejoint la faculté de médecine de Montpellier à une date inconnue avant d'être récupérée par l'établissement, dans un état ne permettant plus sa présentation au public. La sculpture a été restaurée en 2015 : le bras droit du personnage, maintenu à l'aide d'un système de clefs, s'était désolidarisé du reste du corps mais a pu être réinstallé. D'autre part, la surface de L'Écorché portait des inscriptions, précisant le nom de chaque muscle. Pour conserver la mémoire de cette vocation pédagogique et scientifique et la rendre directement visible au visiteur, le choix a été fait de les conserver. L'œuvre est désormais présentée dans la belle série d'académies d'hommes peints par Fabre lors de ses années de résidence à l'Académie de France à Rome, de 1788 à $1790^{31}$, et avec celle peinte en 1778 par David, surnommée Hector $^{32}$. Ce bel ensemble permet d'évoquer auprès des visiteurs la formation des jeunes artistes à la fin du XviII siècle, en soulignant que c'est devant L'Écorché de Houdon que le jeune Fabre, élève de l'école de dessin de Montpellier, a reçu ses premières leçons.

Figure 5

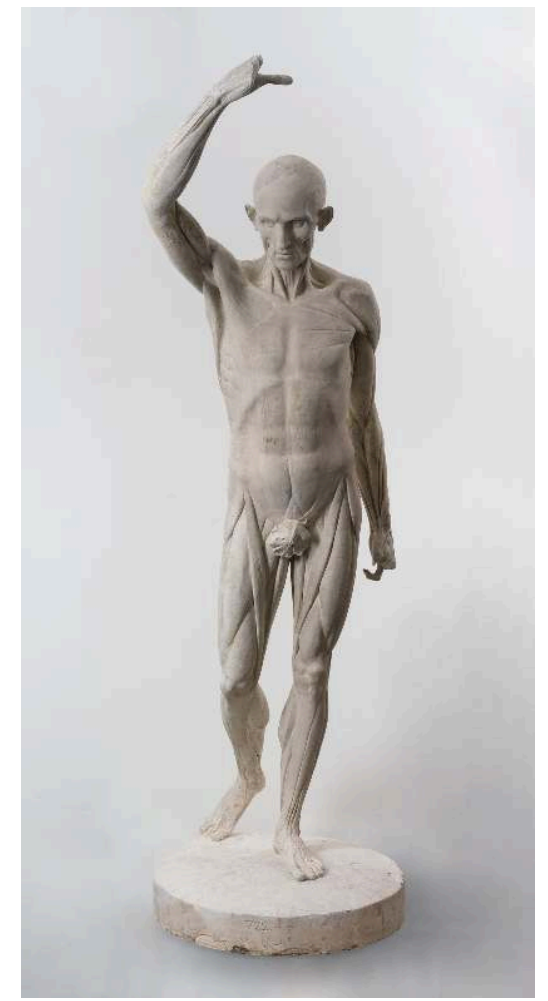

Écorché au bras relevé, Jean-Antoine Houdon, vers 1778, plâtre conservé au musée Fabre (inv. 806.32), Montpellier.

(C) Photo Frédéric Jaulmes, 2017 (Montpellier - Méditerranée Métropole)

Le tirage en plâtre du buste de Jean Deydé [fig. 6], mentionné dès 1787 dans la collection de modèles de la Société des beaux-arts, a également été conservé. Il s'agit d'un moulage tiré d'après le buste en marbre de ce conseiller à la Cour des comptes de Montpellier, sculpté en 1684 par Christophe Veyrier ${ }^{33}$ (1637-1689), élève, collaborateur et neveu par alliance de Pierre Puget (1620-1694) qui conçut le décor de la chapelle funéraire des Deydé dans la cathédrale de Montpellier. Le plâtre a sans doute été offert 
par l'arrière-petit-fils de Jean Deydé, Bernard Daniel Deydé, membre fondateur de la Société des beaux-arts, connu sous le nom de «chevalier Deydé ». Il est fort probable que ce tirage en plâtre ait été exécuté par Jean-Louis Journet (vers 1730 - ?), sculpteur originaire du Vigan qui, après une carrière parisienne et danoise aux côtés de Jacques François Saly (1717-1776), revint en Languedoc, s'installa à Montpellier et devint l'un des professeurs de l'école de dessin de la Société des beaux-arts ${ }^{34}$.

\section{Figure 6}

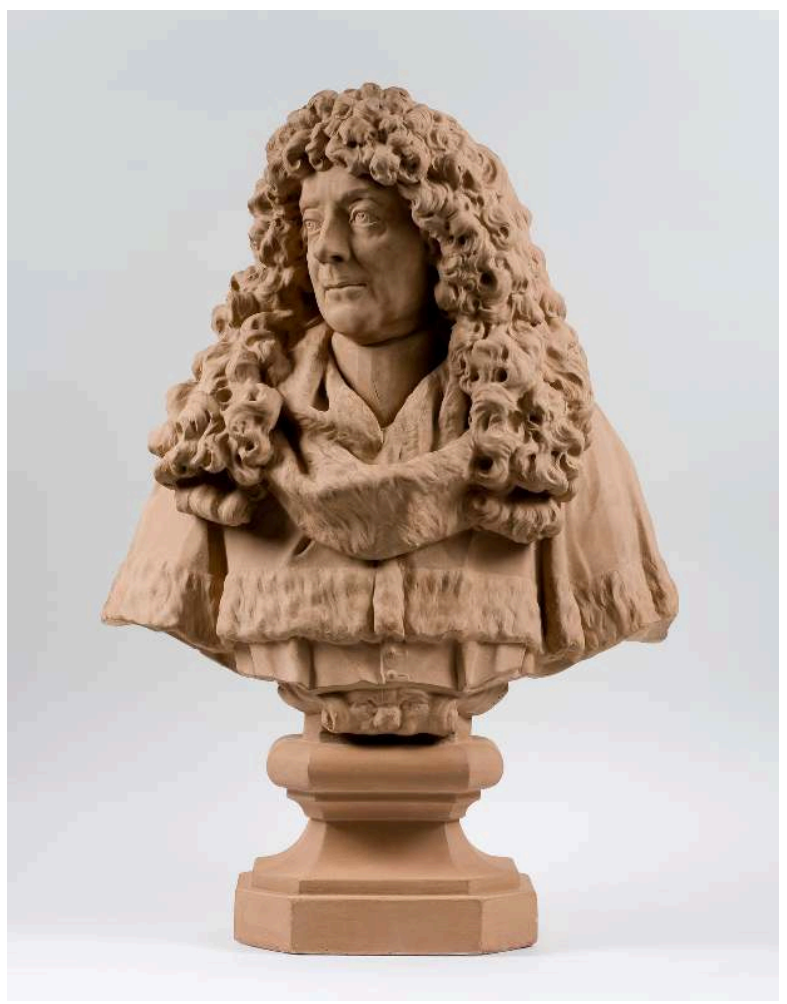

Jean Deydé (1617-1687), attribué à Jean-Louis Journet, d'après Christophe Veyrier, plâtre conservé au musée Fabre (inv. 806.34), Montpellier.

(c) Photo Frédéric Jaulmes, 2017 (Montpellier - Méditerranée Métropole)

De la collection Philippe-Laurent de Joubert, acquise en 1795 par le directoire du département de l'Hérault, aucun plâtre n'est hélas aujourd'hui localisé. Ne demeurent aujourd'hui que les quelques sculptures « en dur » de cet ensemble. Trois bas-reliefs en marbre, d'après des sculptures antiques, visiblement exécutés par deux mains différentes, ont pu être préservés, et sont peut-être, pour deux d'entre eux, l'œuvre de Journet $^{35}$ [fig. 7, fig. 8, fig. 9]. Un quatrième bas-relief, en terre cuite, représentant des amours, évoque davantage l'esprit des sculptures de Clodion ${ }^{36}$. 
Figure 7

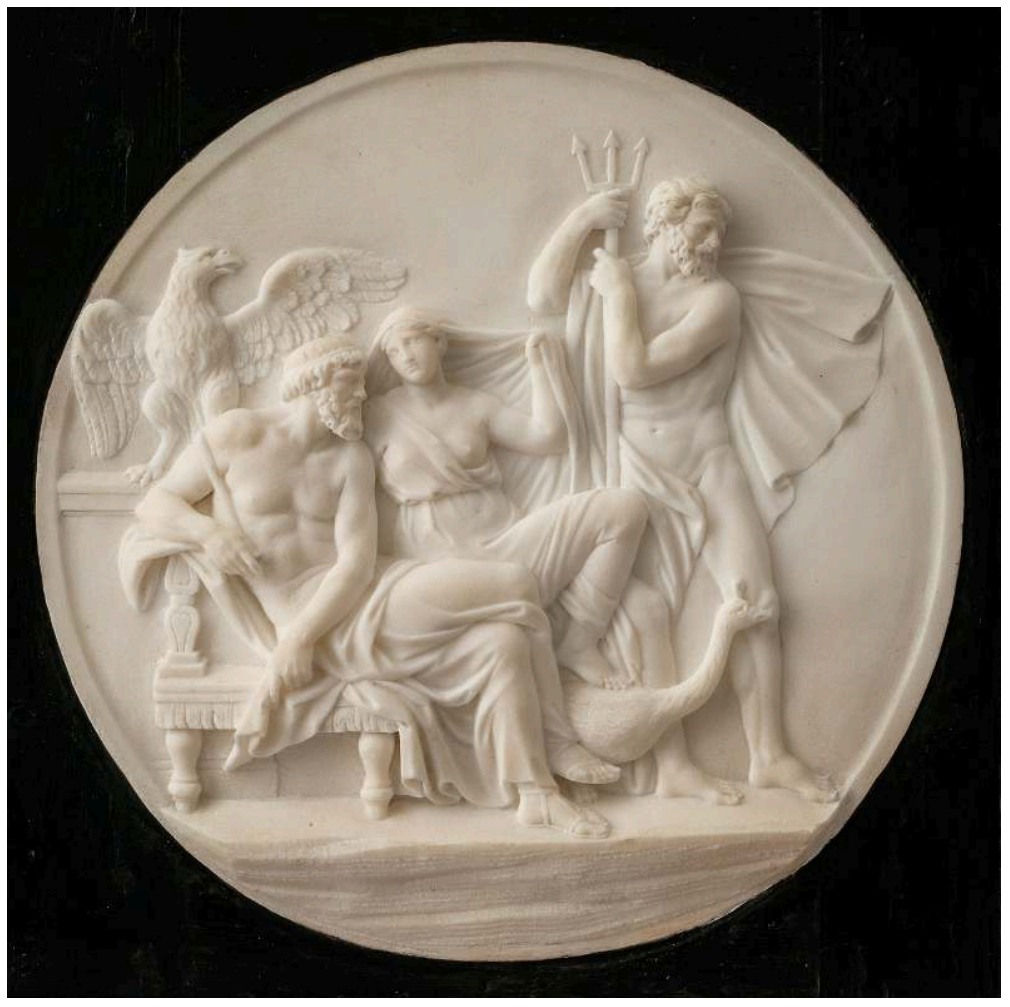

Jupiter, Neptune et Junon, attribué à Jean-Louis Journet, d'après l'antique, marbre conservé au musée Fabre (inv. 806.35), Montpellier.

(c) Photo Frédéric Jaulmes, 2017 (Montpellier - Méditerranée Métropole). 
Figure 8

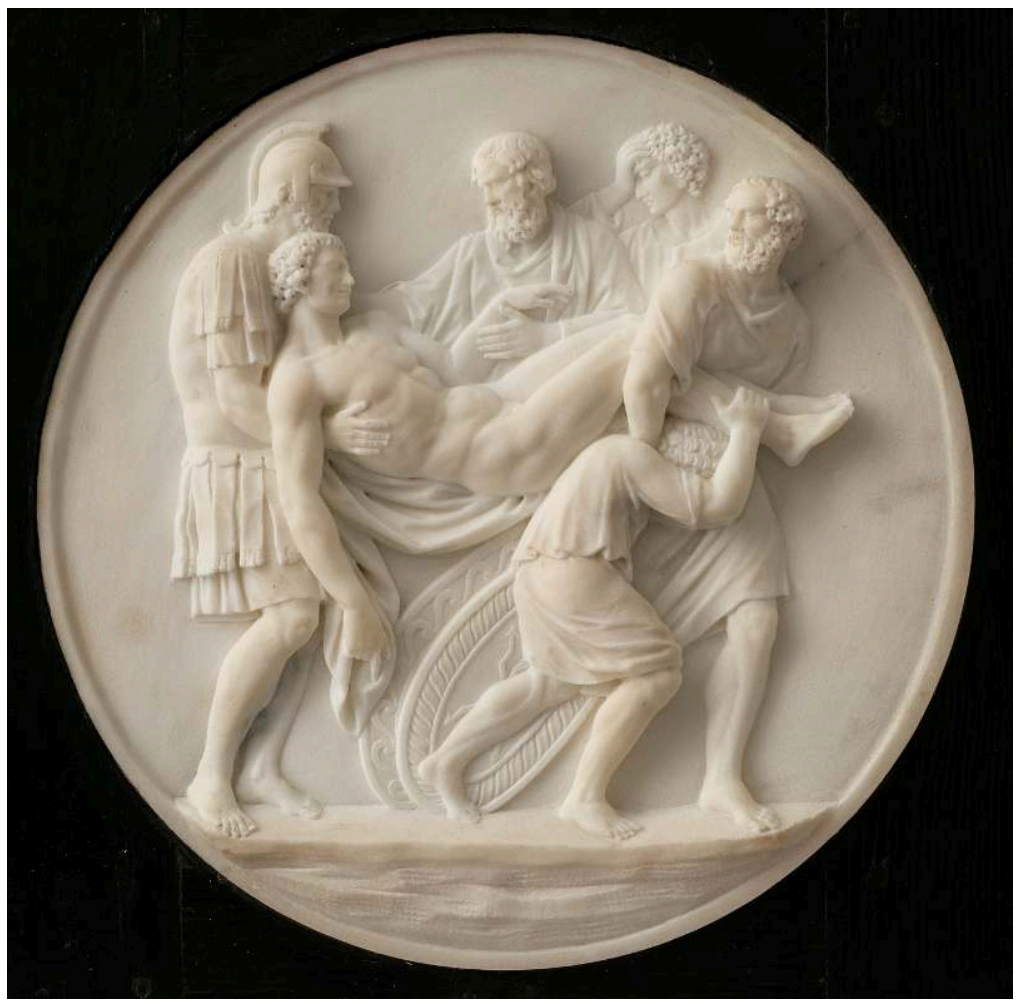

La Mort de Méléagre, attribué à Jean-Louis Journet, d'après l'antique, marbre, conservé au musée Fabre (inv. 806.36), Montpellier.

(c) Photo Frédéric Jaulmes, 2017 (Montpellier - Méditerranée Métropole).

Figure 9

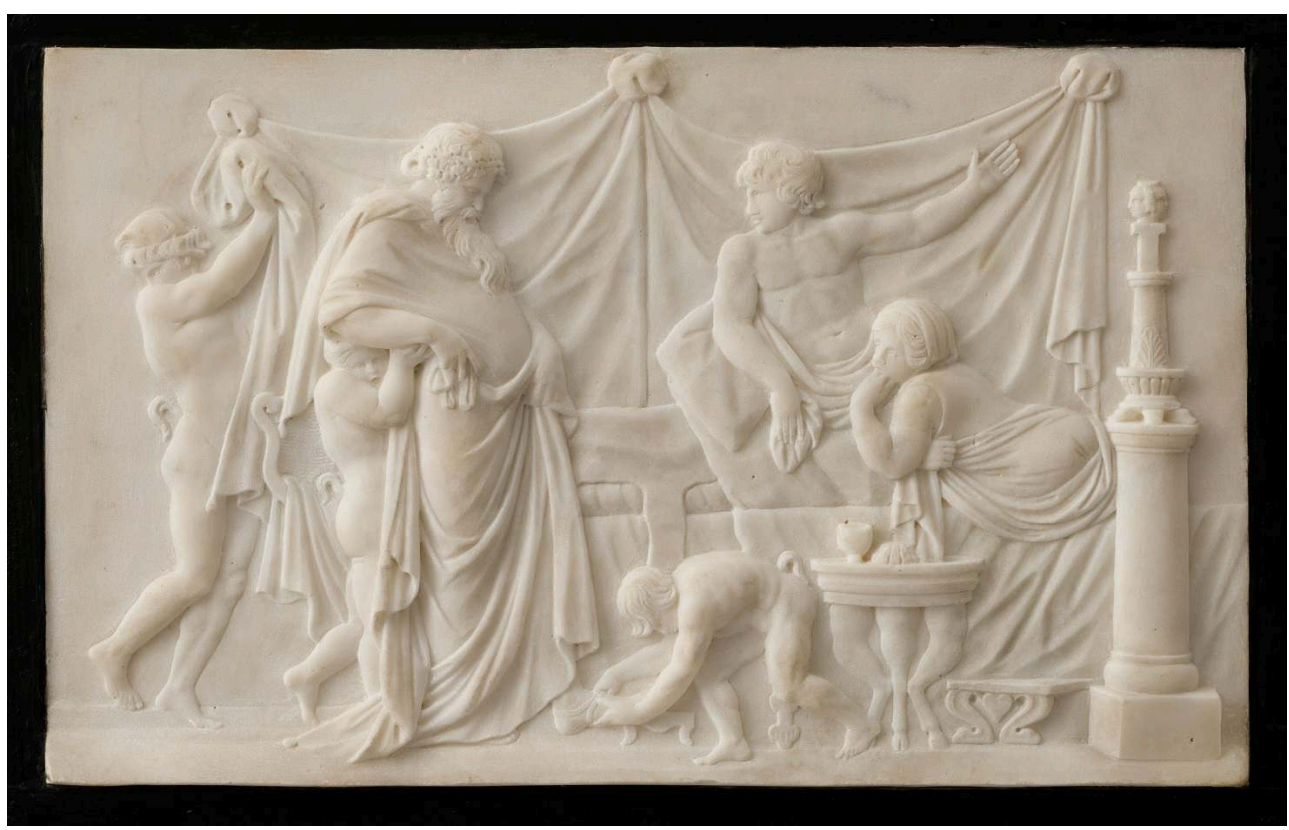

Un poète recevant le dieu du vin Dionysos, sculpteur anonyme du xvıI siècle, d'après l'antique, marbre conservé au musée Fabre (inv. 806.37), Montpellier.

(c) Photo Frédéric Jaulmes, 2017 (Montpellier - Méditerranée Métropole) 
Enfin, pour ce qui est des modèles dessinés ou gravés, aucune œuvre n'a pu être à ce jour repérée. Cependant, un ensemble de dessins présentés par des élèves pour le prix annuel de la Société des beaux-arts a été identifié dans les fonds des archives départementales de l'Hérault ${ }^{37}$. Ils s'inspirent pour deux d'entre eux d'un modèle unique, repris de la fresque du Dominiquin (1581-1641) Sainte Cécile distribuant ses biens aux pauvres (1616-1617, Rome, église Saint-Louis-des-Français), et provient sans doute de la collection de modèles de l'école de dessin de Montpellier.

La moisson s'avère donc bien maigre aujourd'hui mais de futures recherches dans les différents fonds patrimoniaux de la ville pourront sans doute permettre d'identifier d'autres fragments de cette collection oubliée. Cette histoire effacée a cependant pu être présentée au public en 2017-2018, lors de l'exposition «Le musée avant le musée. La Société des beaux-arts de Montpellier ». La mise en valeur de cette histoire illustre le défi plus ambitieux de dessiner une histoire des collections et des anciennes provenances au sein d'un parcours dont l'objectif est aussi de raconter l'histoire de l'évolution chronologique des formes plastiques, de salle en salle.

\section{NOTES}

1. Voir notamment HILAIRE Michel, «Le musée Fabre, une passion de collectionneurs ", in AMIC Sylvain, FARIGOULE Jérôme, HILAIRE Michel \& ZEDER Olivier, Guide musée Fabre, Paris, Réunion des musées nationaux, 2006, p. 7-17.

2. Sur cette institution et son histoire, voir STEIN Henri, «La Société des beaux-arts de Montpellier ", in [s.n.], Mélanges offerts à Henry Lemonnier [...] par la Société de l'histoire de l'art français, ses amis et ses élèves, Paris, Édouard Champion, coll. « Archives de l'art français ", 1913 ; NICOLAS Fabien, «Un exemple de la vie artistique en province à la fin du XVIII ${ }^{\mathrm{e}}$ siècle : la Société des Beaux-Arts de Montpellier (1779-1787) ", mémoire de maîtrise d'histoire de l'art soutenue à l'université Paul-Valéry Montpellier-III sous la direction de Laure Pellicer (Montpellier, 1998) ; TRANI Elsa, «De la Société des beaux-arts au musée municipal, une histoire de la culture artistique montpelliéraine à la fin de l'Ancien Régime ", in HILAIRE Michel \& STÉPANOFF Pierre (dir.), Le Musée avant le musée. La Société des beaux-arts de Montpellier (1779-1787), catalogue d'exposition (Montpellier, musée Fabre, 9 décembre 2017-11 mars 2018), Gand/ Montpellier, Snoeck / musée Fabre, 2017, p. 29-47.

3. Sur ce personnage, voir ANDRÉ Pascale, « Abraham Fontanel, marchand d'art et collectionneur (1741-1817) », mémoire de DEA d'histoire de l'art soutenu à l'université Paul-Valéry MontpellierII I sous la direction de Laure Pellicer (Montpellier, 2001) ; ANDRÉ-PONS Pascale, « Les multiples talents de M. Fontanel », in BERLAN Hélène, CHAPRON Emmanuelle, LUCIANI Isabelle \& LE THIEC Guy (dir.), Érudits, collectionneurs et amateurs. France méridionale et Italie (XVIII ${ }^{e}-\mathrm{XIX} \mathrm{X}^{e}$ siècle), Aix-enProvence, Presses universitaires de Provence, coll. «Le temps de l'histoire ", 2017, p. 229-237 ; STÉPANOFF Pierre, "Abraham Fontanel (1741-1817), portrait d'un homme zélé », in HILAIRE Michel \& STÉPANOFF Pierre (dir.), Le Musée avant le musée, p. 15-27.

4. Sur les œuvres présentées dans les différents salons montpelliérains de 1779 à 1786, voir STÉPANOFF Pierre. "Catalogue sommaire», in HILAIRE Michel \& STÉPANOFF Pierre (dir.), Le Musée avant le musée, p. 126-188. 
5. Voir à ce sujet LAHALLE Agnès, Les Écoles de dessin au XVIII siècle. Entre arts libéraux et arts mécaniques, Rennes, Presses universitaires de Rennes, coll. « Histoire », 2006, p. 221-231.

6. «Registre contenant les séances et les délibérations de la Société des beaux-arts de Montpellier ", 1779-1787, p. 8-9 (Montpellier, médiathèque centrale Émile-Zola, Ms 247).

7. Voir HILAIRE Michel \& STÉPANOFF Pierre (dir.), Le Musée avant le musée, notice $\mathrm{n}^{\circ} 18$, p. 102-103. Contrairement à ce que nous affirmions dans ce catalogue, l'Écorché envoyé par Houdon en 1779 ne peut se confondre avec celui «au bras relevé » aujourd'hui conservé au musée Fabre, car le modèle de ce dernier a été élaboré par le sculpteur en 1790. Voir à ce sujet COMAR Philippe (dir.), Figures du corps. Une leçon d'anatomie à l'École des beaux-arts, catalogue d'exposition (Paris, École nationale supérieure des beaux-arts, 21 octobre 2008-4 janvier 2009), Paris, Éditions des Beaux-arts de Paris, 2008, n 68-72, p. 206-214. La date d'arrivée au musée Fabre du modèle au bras levé n'est pas connue, mais il est peut-être possible de l'identifier avec le second Écorché mentionné dans l'inventaire du Musée municipal rédigé par Jacques Bestieu en 1806 ( « Catalogue des objets d'arts renfermés dans le musée de la ville de Montpellier dressé par Jacques Bestieu le 22 octobre 1806 », archives municipales de Montpellier, Ms. 2R17, f. 7).

8. Voir HILAIRE Michel \& STÉPANOFF Pierre (dir.), Le Musée avant le musée, notice $n^{\circ}$ 9, p. 90-91.

9. Lettre de Jean-Antoine Houdon à Abraham Fontanel du 4 décembre 1778 (archives départementales de l'Hérault (ADH), D 233).

10. «Registre contenant les séances et les délibérations... », p. 20.

11. "Registre contenant les séances et les délibérations... ", p. 52.

12. FONTANEL Abraham, « Mémoire concernant l'origine et l'établissement d'une école de dessin dans la ville de Montpellier, 30 floréal an IV [19 mai 1796]» (ADH, L 2495), reproduit dans HILAIRE Michel \& STÉPANOFF Pierre (dir.), Le Musée avant le musée, p. 206-209.

13. «Inventaire des effets cédés à la province de Languedoc par délibération de MM. les Associés fondateur de la Société des beaux-arts de Montpellier, dressé le 19 février 1787 par Abraham Fontanel » (ADH, C 7903).

14. Voir à ce sujet CHEVALIER Alain, HILAIRE Michel \& STÉPANOFF Pierre (dir.), De marbre blanc et de couleur. La chapelle Deydé de la cathédrale de Montpellier, catalogue d'exposition (Montpellier, musée Fabre, 16 novembre-15 mars 2020), Gand / Montpellier, Snoeck / musée Fabre, 2020, fig. 1, p. $24, \mathrm{n}^{\circ} 17$, p. $232-236$.

15. Au sujet de ce personnage, voir ALEX Barbara, « Recherches sur la vie et l'œuvre du peintre montpelliérain Jacques Bestieu (1754-1842)», mémoire de maîtrise d'histoire de l'art sous la direction de Laure Pellicer, soutenu à l'université Paul-Valéry Montpellier-III (Montpellier, 1998). Voir également HILAIRE Michel \& STÉPANOFF Pierre (dir.), Le Musée avant le musée, p. 214.

16. Au sujet de ce personnage, voir CROUX-FAVRE Brigitte, "Philippe-Laurent de Joubert, 1729-1792, trésorier de la Bourse des États du Languedoc. Ses portraits, son cabinet de curiosités, ses collections ", mémoire de maîtrise d'histoire de l'art sous la direction de Laure Pellicer, soutenu à l'université Paul-Valéry Montpellier-III (Montpellier, 1986). Voir également HILAIRE Michel \& STÉPANOFF Pierre (dir.), Le Musée avant le musée, p. 218.

17. «Inventaire des plâtres moulés d'après l'antique appartenant à feu Joubert, dressé par Abraham Fontanel » (ADH, L 2098).

18. «Inventaire des objets confiés au conservateur des dessins près l'école centrale du 23 frimaire an VI [13 décembre 1797)", dressé par Abraham Fontanel et Claude Daumas (ADH, L 2483).

19. «Registre contenant les séances...», p. 63-64.

20. Montpellier, musée Fabre (inv. 806.11), voir HILAIRE Michel \& STÉPANOFF Pierre (dir.), Le Musée avant le musée, notice $\mathrm{n}^{\circ} 7, \mathrm{p} .86-87$.

21. Montpellier, musée Fabre (inv. 806.12), voir HILAIRE Michel \& STÉPANOFF Pierre (dir.), Le Musée avant le musée, notice $\mathrm{n}^{\circ} 28, \mathrm{p} .116-117$. 
22. «Catalogue des objets d'arts renfermés dans le musée de la ville de Montpellier dressé par Jacques Bestieu le 22 octobre 1806 ", archives municipales de Montpellier, Ms. 2R17, f. 7.

23. FABRE François-Xavier, Notice des tableaux et autres objets exposés au musée Fabre de Montpellier, Montpellier, [s. n.], 1828 [réed. 1830], notices n 346 «Argus endormi », n 347 « Mercure épie le moment où Argus est endormi [...], ces deux statues sont de Mr. de Bay père, qui les a données à la ville ", n 348 « Eurydice piquée par un serpent sur les bords du Pénée ", n 349 "Silène ivre [...], ces deux statues ont été exécutées en marbre par Mr. Legendre-Héral, de Montpellier [...], il les a données au Musée ». Ces quatre plâtres sont aujourd'hui perdus.

24. «Inventaire général des tableaux, dessins, gravures, bronzes, marbres, plâtres et autres objets d'art existant dans le Musée Fabre de la ville de Montpellier. Le dit inventaire dressé en 1838 par Casimir Desmazes, d'après l'ordre de M. le Maire de Montpellier », p. 114 (Montpellier, musée Fabre, non coté).

25. Montpellier, musée Fabre (inv. D45.2.1). Voir HILAIRE Michel \& STÉPANOFF Pierre (dir.), Le Musée avant le musée, notice $\mathrm{n}^{\circ}$ 47, p. 148.

26. Montpellier, musée Fabre (inv. 874.1.1). Voir HILAIRE Michel \& STÉPANOFF Pierre (dir.), Le Musée avant le musée, notice $\mathrm{n}^{\circ} 17, \mathrm{p} .100-101$.

27. Montpellier, musée Fabre (inv. D45.1.1 et D45.1.2). Voir HILAIRE Michel \& STÉPANOFF Pierre (dir.), Le Musée avant le musée, notice $\mathrm{n}^{\circ}$ 45-46, p. 147.

28. Montpellier, musée Fabre (inv. 828.5.1 et 825.1.2). Voir BÜCKLING Maraike \& SCHERF Guilhem (dir.), Jean-Antoine Houdon. La sculpture sensible, catalogue d'exposition (Francfort, Liebieghaus Skulpturensammlung, 29 octobre 2009-28 février 2010; Montpellier, musée Fabre, 17 mars-27 juin 2010), Paris, Somogy, notices $\mathrm{n}^{\text {os }} 1-2$, p. 66-71.

29. Montpellier, musée Fabre (inv. 95.6.1). Voir BÜCKLING Maraike \& SCHERF Guilhem (dir.), Jean-Antoine Houdon..., notice $\mathrm{n}^{\circ} 4$, p. 72-73.

30. Voir note 7.

31. Montpellier, musée Fabre, Soldat romain au repos (inv. 825.1.63), Le Repos du gladiateur (inv. 2005.2.1), Saint Sébastien (inv. 825.1.62), Abel expirant (inv. 825.1.60). Voir à ce sujet HILAIRE Michel \& PELLICER Laure, François-Xavier Fabre (1766-1837). De Florence à Montpellier, catalogue d'exposition (Montpellier, musée Fabre, 14 novembre 2007-24 février 2008 ; Turin, Galleria civica d'arte moderna e contemporanea, 11 mars-2 juin 2008), Paris / Montpellier, Somogy / musée Fabre, 2008, n 14-17, p. 112-115.

32. Montpellier, musée Fabre (inv. 851.1.1).

33. New York, the Metropolitan Museum of Art (inv. 66.129a et b). Voir à ce sujet CHEVALIER Alain, HILAIRE Michel \& STÉPANOFF Pierre (dir.), De marbre blanc et de couleur..., notice $\mathrm{n}^{\circ} 16$, p. 227-231.

34. Voir à ce sujet ANATOLE Christian, «De Copenhague à Montpellier, un sculpteur languedocien du XVIII ${ }^{\mathrm{e}}$ : J. Journet », Ménestrel, nº 6, 1975, p. 18-22; HILAIRE Michel \& STÉPANOFF Pierre (dir.), Le Musée avant le musée, p. 218.

35. Montpellier, musée Fabre (inv. 806.35, 806.36, 806.37). Voir HILAIRE Michel \& STÉPANOFF Pierre (dir.), Le Musée avant le musée, notice n 29-30, p. 118-119.

36. Montpellier, musée Fabre (inv. 806.38).

37. ADH (1 Fi, 1037, 1038, 1039, 1040, 1041). Voir HILAIRE Michel \& STÉPANOFF Pierre (dir.), Le Musée avant le musée, notices $\mathrm{n}^{\text {os }} 22-24$, p. 108-111. 


\section{RÉSUMÉS}

Bien que fondé en 1825 par François-Xavier Fabre, le musée Fabre de Montpellier possède une origine plus ancienne. Dès 1779, une école gratuite de dessin avait été créée dans la ville languedocienne. Au fil des années et de ses multiples métamorphoses, jusqu'à la création du musée en 1795 , une importante collection de modèles avait été constituée et mise à disposition des élèves. Malheureusement, ce matériel à vocation pédagogique a peu à peu disparu des collections, pour laisser la place à une conception plus strictement patrimoniale et artistique des collections. De récentes recherches et la tenue d'une exposition sur ce sujet au musée Fabre en 2017-2018 permettent aujourd'hui de mieux percevoir cette histoire et de mieux comprendre la genèse du musée de Montpellier, à la fin de l'Ancien Régime et sous la Révolution. Elles soulignent l'ambiguité de la définition de ce premier musée, entre instruction et délectation.

If the Musée Fabre has been created in 1825 by the painter François-Xavier Fabre, the Fabre museum of Montpellier has an older origin. In the year 1779, a free drawing school was founded in the southern french city. Over the years and after its multiple metamorphoses, until the museum's creation in 1795, a large collection of models was assembled and made available to students. Unfortunately, this pedagogical equipment has gradually disappeared to give way to a more strictly patrimonial and artistic conception of the collections. Recent research and the organization of an exhibition on this subject at the musée Fabre in 2017-2018 now allow us to perceive this history, as well as to understand the genesis of the museum of Montpellier, at the end of the Ancien Regime and during the French Revolution. It underlines the ambiguity of the definition of this first museum, between instruction and pleasure.

INDEX

Keywords : Montpellier, Musée Fabre, Fabre (François-Xavier), drawing school, French Revolution, Society of Fine Arts, copy, pedagogy, cast, plaster, models

Mots-clés : Montpellier, musée Fabre, Fabre (François-Xavier), école de dessin, Révolution française, Société des beaux-arts, copie, pédagogie, moulages, plâtres, modèles

\section{AUTEUR}

\section{PIERRE STÉPANOFF}

Conservateur du patrimoine, musée Fabre, Montpellier

p.stepanoff@montpellier3m.fr 